\title{
High prevalence of HIV infection and unprotected anal intercourse among older men who have sex with men in China: a systematic review and meta-analysis
}

Yong-ze Li ${ }^{1,2+}$, Jun-jie X $u^{1,2+}$, Han-zhu Qian ${ }^{3,4}$, Bing-xin You ${ }^{5}$, Jing Zhang ${ }^{1,2}$, Jian-ming Zhang ${ }^{6}$, Qing-hai Hu ${ }^{1,2}$, Zhen-xing Chu ${ }^{1,2}$, Shu-yang Liu ${ }^{1,2}$, Yong-jun Jiang ${ }^{1,2}$, Wen-qing Geng ${ }^{1,2}$ and Hong Shang ${ }^{1,2^{*}}$

\begin{abstract}
Background: China has the largest number of the elderly in the world. As the proportion of elderly is rapidly increasing among national reported HIV/AIDS cases, it is a concern about HIV epidemic among older MSM in China. However, studies on HIV prevalence and unprotected anal intercourse (UAI) among Chinese older MSM were relatively few or generally had small sample sizes.

Methods: English and Chinese articles published in peer-reviewed journals were identified by systematically searching 5 electronic databases including PubMed and through cross-referencing. Summary prevalence rates of HIV infection and UAI with male sexual partners were calculated, and analyses were performed using the software Comprehensive Meta-Analysis V2.0 and SPSS V17.0. Subgroup analyses were performed separately by sample size, study year, study location, recruitment settings and sampling method.

Results: Twenty eligible cross-sectional studies (3 in English and 17 in Chinese), published between 2005 and 2013, with a total of 2812 older MSM participants, were identified. Our meta-analyses showed that the prevalence of HIV, syphilis and UAI in the last 6 months were 11.6\% (95\% confidence interval [CI]: 8.0\%-16.6\%), 23.0\% (95\% Cl: 15.8\%-32.3\%) and 79.5\% (95\% Cl: $72.7 \%-84.9 \%)$, respectively. HIV prevalence increased over the study period (6.3\% in 2003-2007; $8.6 \%$ in 2008-2009, and $11.5 \%$ in 2010-2011, trend test Chi-square $=7.02, p=0.008$ ). The pooled prevalence of HIV $(11.6 \%$ vs. $5.2 \%$, Chi-square value $=36.2, p<0.001)$ and UAI (79.5\% vs. $52.6 \%$, Chi-square value $=440.04, p<0.001)$ among older MSM were both significantly higher than among younger age group (age < 50 years).

Conclusions: Older Chinese MSM have high prevalence of HIV and syphilis. Unprotected anal sex is common and further puts them at high risks of acquiring and transmitting HIV, which was one of reasons for the rapid increasing of national reported older male HIV/AIDS cases. Prevention intervention programs should be specially tailored for this high risk MSM subgroup.
\end{abstract}

Keywords: HIV, Syphilis, Unprotected anal intercourse (UAI), Men who have sex with men (MSM), Older MSM, China

\footnotetext{
* Correspondence: hongshang100@hotmail.com

${ }^{\dagger}$ Equal contributors

'Key Laboratory of AIDS Immunology of National Health and Family Planning

Commission, Department of Laboratory Medicine, The First Affiliated

Hospital, China Medical University, Shenyang, Liaoning, China

${ }^{2}$ Collaborative Innovation Center for Diagnosis and Treatment of Infectious

Diseases, Hangzhou, China

Full list of author information is available at the end of the article
} 


\section{Background}

Human immunodeficiency virus (HIV) infection and other sexually transmitted infections (STIs) continue to disproportionately affect men who have sex with men (MSM) in China [1]. A study conducted in 61 cities in China between February 2008 to September 2009 found that 4.9\% of MSM were infected with HIV [2]. According to a report from the Chinese Ministry of Health, it estimated that there were approximately 780,000 people living with HIV/ AIDS in 2011 in China [3], 17.4\% of the estimated HIV/ AIDS cases were attributable to male-to-male sexual contact. Sentinel surveillance data have shown an increasing trend of HIV infection among MSM, from 2.0\% in 2007 to $6.3 \%$ in 2011 [4].

China has the largest number of the elderly in the world [5]. According to a report from the China National Committee on Ageing, there were an estimated 185 million elderly people (aged 60 years or older) in China by the end of 2011, and about half are men [6]. Chinese elderly people were generally not considered as a population at high-risk for HIV infection [7]. Whereas, among nationally reported HIV/ AIDS cases, the proportion of total cases accounted for by the $50-64$ age group increased from $1.6 \%$ to $13.8 \%$ in 2000 and 2011, respectively [4].

As the number of new HIV cases amongst the 50+ age group increased noticeably in recent years, there have been a few studies exploring the reasons in recent years, and shown that engaging in commercial sexual activities after their retirement is a major factor for the increased HIV epidemic among the older men [8,9]. As MSM has become a major at-risk group, it is a concern about HIV among older MSM in China. However, studies on HIV prevalence and unprotected anal intercourse (UAI) among Chinese older MSM are relatively few and generally have small sample sizes $[7,10,11]$; it will be helpful for assessing the magnitude of HIV epidemic and risk behaviors in this age group through summarizing the findings from these studies. We performed Meta analysis of the prevalence of HIV, syphilis and unprotected anal sex (UAI) in the last 6 months among older MSM in China.

\section{Methods}

\section{Search strategy}

Studies published in English or Chinese were identified through searching the following electronic databases: PubMed, China National Knowledge Infrastructure (CNKI), Chinese Scientific Journals Full text Database (CQVIP), Wanfang and Google Scholar. The keywords and medical subject headings "HIV", "AIDS", "MSM", "gay", "homosexual", "elderly", "older", "aged”, "old”, and "China” were used to search for potentially relevant studies (e.g. http://www. ncbi.nlm.nih.gov/pubmed/?term=old+MSM+HIV+China). The last date of search was July 1st 2013. This meta- analysis was conducted in accordance with the guidelines of the Preferred Reporting Items for Systematic Reviews and Meta-Analyses statement (PRISMA) [12].

\section{Inclusion and exclusion criteria}

Studies were eligible for inclusion in the review if they met the following criteria: (1) published in Chinese or English language; (2) conducted in China; (3) study participants aged $\geq 50$ years old MSM; (4) reported homosexual behaviors (recall window $\leq 6$ months); (5) presented laboratory diagnosis of HIV and/or syphilis, as well UAI which was defined as no condom use in insertive or receptive anal sex for at least once in the past 6 months.

Studies were excluded if they met the following criteria: (1) not an original study, e.g., review or editorial; (2) not a peer review journal article, e.g., government report or conference abstract; (3) if the same study data were published in both English and Chinese, Chinese publications were excluded from the review, and (4) studies that exclusively recruited MSM participants with specific behaviors that may inflate the estimation of the prevalence of HIV or syphilis or UAI, e.g., money boys, illegal drug users.

\section{Data extraction and study quality assessment}

For all eligible studies, two reviewers (YL and BY) independently extracted the following data from original publications: first author and year of publication; study location and study year; sampling method and recruitment setting; sample size; and results of HIV and syphilis tests and UAI prevalence (if available). Disagreements between the two reviewers during data extraction were reconciled by a third reviewer (JX).

The quality assessment checklist for observational studies (QATSO score) was selected to evaluate the quality of the included studies [13]. Items were scored as 1, 0, NA, which corresponded to "yes", "no", or "not applicable", respectively. The total score was divided by the total number of all applicable items. Values between 0\%- 33\%, 34\%-66\%, and 67\%- 100\% represent "bad", "satisfactory" and "good" quality, respectively [13].

\section{Statistical analysis}

Standard meta-analytic approaches were used in calculating prevalence estimates for individual studies and for aggregating estimates across studies [14]. Meta-analysis of the prevalence of HIV and syphilis infections and UAI was carried out using the Comprehensive Meta-Analysis software (V2.0, Biostat, Englewood, NJ). The pooled prevalence was calculated using the inverse variance method. The average effect size across all studies is computed as a weighted mean, whereby the weights are equal to the inverse variance of each study's effect estimator [15]. Larger 
studies and studies with less random variation are given greater weight than smaller studies [15].

We also tested the heterogeneity of the findings across studies by using the $\mathrm{Q}$ statistic. The aggregated results were calculated based on a random-effects model, which provides a more conservative estimate of variance and generates more accurate inferences about a population of studies beyond those included in this review. The Begg rank correlation method was used to assess the potential for publication bias ( $\mathrm{p}<0.05$ was considered the indicative of statistically significant publication bias). Analyses were conducted separately for HIV, syphilis and UAI.

Stratified analyses were conducted to examine whether the prevalence of HIV infection differed by study location, study year, sample size, recruitment setting and sampling method. The changes of HIV prevalence over the study period and the difference between the pooled prevalence of HIV and UAI among older MSM and their younger counterparts were analyzed for linear trend using SPSS (V17.0, Chicago, IL, USA). Their younger counterparts were defined as the total MSM minus the older MSM within the studies.

In the sensitivity analyses, we compared the overall estimate with the estimates obtained after iterations using $\mathrm{k}-1$ studies $(\mathrm{k}=$ number of independent samples). We removed a study and calculated the overall prevalence estimate. Then, we replaced that study, removed another, and repeated the process.

\section{Results}

\section{Study selection}

A total of 3657 citations were identified, of which 68 potentially relevant articles were selected for further screening, and eventually 20 articles (3 published in English and 17 in Chinese) with 2812 older MSM participants met the inclusion criteria (Figure 1). Besides, we identified 59556 younger MSM (age $<50$ years) as their comparison. Table 1 provides a descriptive summary of these studies [2,16-34]. All studies had laboratory testing results of HIV infection, 7 had results of syphilis infection, and 11 measured UAI. All the included studies met the criteria of "good quality" (values between $67 \%$ and 100\%) (Additional file 1).

\section{Prevalence of HIV and syphilis infection}

Figure 2 shows the summarized estimates of HIV prevalence among older MSM from 2003-2011 (11.6\%; 95\% confidence interval $[\mathrm{CI}]: 8.0 \%-16.6 \%)$ with the prevalence rate in individual studies ranging from $2.1 \%$ (95\% CI: 0.1\%-25.9\%) to $43.8 \%$ (95\% CI: $27.9 \%-61.0 \%$ ). The summary HIV prevalence among older MSM was significantly higher than their younger counterparts $(11.9 \%$ vs. $5.1 \%$, Chi-square value $=40.3, \mathrm{p}<0.001)$. No significant publication bias was observed $(p=0.77)$. However, there was

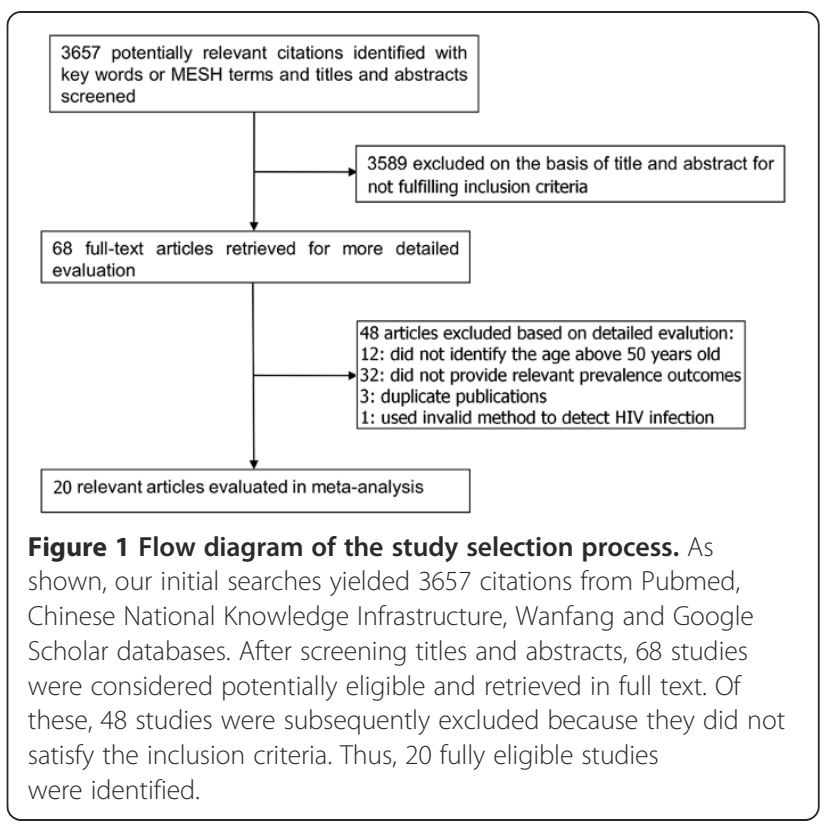

substantial heterogeneity between studies ( $\mathrm{p}$ for $\mathrm{Q}$ test, $\mathrm{p}<0.01 ; I^{2}=76.51$ ).

Figure 3 shows the summarized prevalence of syphilis infection, of the average prevalence was $23.0 \%$ (95\% CI: $15.8 \%-32.3 \%$ ) with a range from $2.3 \%$ (95\% CI: $0.1 \%$ $7.7 \%$ ) to $41.9 \%$ (95\% CI: $28.2 \%-56.9 \%$ ). The summary prevalence of syphilis infection among older MSM was marginal significantly higher than their younger counterparts $(23.0 \%$ vs. $10.0 \%$, Chi-square value $=3.57, \mathrm{p}=0.06)$. There was medium heterogeneity between studies $(\mathrm{p}=$ $\left.0.03 ; I^{2}=58.59\right)$. No publication bias was observed as assessed by the Begg rank correlation analysis $(\mathrm{p}=0.37)$.

\section{Stratified analyses and trend test of HIV prevalence}

Table 2 presents the findings of stratified analyses, which were conducted for study location, study year, sample size, recruitment setting and sampling method. Heterogeneity between studies was partly reduced in subgroup analyses. The subgroup of studies with larger sample size $(\mathrm{n}>200)$ had a lower prevalence than smaller studies (7.2\% vs. $13.6 \%)$. More recent studies found a higher prevalence than older studies: $11.5 \%(13 / 113)$ in $2010-$ $2011,8.6 \%(139 / 1616)$ in $2008-2009$ and $6.3 \%(68 / 1083)$ in 2003-2007. The trends over these three time periods are statistically significant (trend test Chi-square value $=$ 7.02, $\mathrm{p}=0.008$ ). The highest HIV prevalence was reported in the studies conducted in southwest China (including the provinces of Yunnan, Guangxi, Sichuan, Guizhou, and the city of Chongqing), and the lowest prevalence was reported from nationwide studies (also see Figure 4). The summary prevalence in the southwest was $16.4 \%$, followed by north China (14.7\%), east China (13.0\%), south China $(12.3 \%)$, west China $(9.5 \%)$, and nationwide $(7.2 \%)$. The 
Table 1 Prevalence of HIV infection among older MSM in China

\begin{tabular}{|c|c|c|c|c|c|c|c|c|c|c|c|c|}
\hline \multirow{2}{*}{$\begin{array}{l}\text { First author, } \\
\text { published year }\end{array}$} & \multicolumn{4}{|c|}{ Study design } & \multicolumn{4}{|c|}{ Older MSM } & \multicolumn{4}{|c|}{ Younger MSM } \\
\hline & Location & Study year & $\begin{array}{l}\text { Sampling } \\
\text { method }\end{array}$ & $\begin{array}{l}\text { Recruitment } \\
\text { settings }\end{array}$ & Sample size & HIV infection ${ }^{a}$ & $\begin{array}{c}\text { Syphilis } \\
\text { infection }^{\text {b }}\end{array}$ & $\overline{\text { UAI }}$ & Sample size & HIV infection & $\begin{array}{c}\text { Syphilis } \\
\text { infection }\end{array}$ & UAI \\
\hline$\overline{M a} A B, 2007$ & Yunnan & 2005 & Mixed $^{c}$ & Medical settings & 8 & 1 & $N R^{d}$ & NR & 70 & 7 & NR & $\overline{N R}$ \\
\hline Xi SJ, 2011 & Hangzhou & 2009-2010 & Snowballing & Multiple gay venues or events & 16 & 1 & NR & NR & 514 & 44 & $N R$ & NR \\
\hline Long QP, 2012 & Hunan & 2011 & Mixed & Multiple gay venues or events & 33 & 7 & $N R$ & 27 & 233 & 36 & NR & 178 \\
\hline Zhou JB, 2012 & Changzhou & 2007 & Snowballing & Multiple gay venues or events & 43 & 12 & 18 & NR & 550 & 69 & 167 & NR \\
\hline Wang ZC, 2012 & Xining & 2011 & Mixed & Medical settings & 21 & 2 & 0 & 17 & 382 & 45 & 40 & 233 \\
\hline Qun He, 2006 & Guangzhou & 2003 & Mixed & Medical settings & 23 & 0 & $N R$ & NR & 201 & 0 & NR & NR \\
\hline Xuan ZB, 2012 & Shanghai & 2010-2011 & Snowballing & Medical settings & 9 & 0 & NR & 7 & 89 & 3 & NR & 64 \\
\hline Chu ZX, 2011 & Shenyang & 2008 & Snowballing & Medical settings & 56 & 4 & 11 & 50 & 2018 & 96 & 234 & 1888 \\
\hline Yan Xiao, 2010 & 20 cities & 2006 & Snowballing & Multiple gay venues or events & 1009 & 55 & NR & 834 & 2961 & 91 & $N R$ & 2034 \\
\hline Wu ZY, 2013 & 61 Cities & 2008- 2009 & $\mathrm{RDS}^{\mathrm{e}}$ & Medical settings & 1182 & 90 & 274 & 813 & 45478 & 2196 & 5226 & 23294 \\
\hline Zhou YQ, 2012 & Shanghai & 2010- 2011 & Mixed & Medical settings & 6 & 1 & 2 & NR & 288 & 17 & 36 & NR \\
\hline Yang LG, 2012 & Fuyang & 2010-2011 & Mixed & Multiple gay venues or events & 2 & 0 & $N R$ & 2 & 263 & 15 & NR & 163 \\
\hline Lan GH, 2009 & Guangxi & 2008 & Snowballing & Medical settings & 41 & 2 & 5 & 28 & 1105 & 18 & 60 & 613 \\
\hline Feng F, 2009 & Haikou & 2008 & Mixed & Medical settings & 4 & 0 & NR & NR & 100 & 2 & NR & NR \\
\hline Zhang FX, 2011 & Suzhou & 2008- 2009 & Mixed & Medical settings & 38 & 2 & NR & 27 & 616 & 56 & $N R$ & 384 \\
\hline Li R, 2010 & Dalian & 2009 & Snowballing & Multiple gay venues or events & 6 & 2 & NR & NR & 396 & 16 & NR & NR \\
\hline Chen Y, 2013 & Guizhou & 2008- 2009 & Mixed & Multiple gay venues or events & 32 & 14 & NR & 27 & 798 & 179 & NR & 513 \\
\hline Ni ZM, 2011 & Hangzhou & 2009 & Mixed & Multiple gay venues or events & 5 & 1 & 1 & NR & 214 & 33 & 43 & NR \\
\hline Xu J, 2010 & 4 cities & 2008 & RDS & Medical settings & 252 & 24 & NR & 214 & 1612 & 101 & NR & 1259 \\
\hline Zheng LX, 2012 & Longyan & 2010- 2011 & Snowballing & Multiple gay venues or events & 26 & 2 & NR & $N R$ & 377 & 13 & NR & NR \\
\hline
\end{tabular}

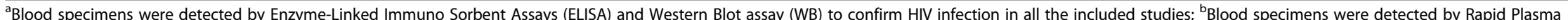
Reagin (RPR) and Treponema Pallidum Particle Agglutination assay (TPPA)to confirm syphilis infection in all the included studies; ${ }^{c}$ Mixed: Snowballing or/and RDS or/and convenience sampling were included; ${ }^{\mathrm{d} N R: \text { Not }}$ reported; ${ }^{\mathrm{e}} \mathrm{RDS}$ : Respond Driven Sampling. 
summary HIV prevalence was significantly higher in studies that recruited participants from gay venues or events than from medical settings (17.4\%, vs. $7.9 \%)$, and was higher in studies using mixed sampling methods $(15.5 \%)$ than using respondent driving sampling (RDS) (8.0\%) and snowball sampling (9.8\%).

\section{Prevalence of unprotected anal intercourse with male partners}

The aggregated findings from 21 studies showed that the prevalence of having ever engaged in UAI with any male partner in the past 6 months was 79.5\% (95\% CI: 72.7\%$84.9 \%$ ) (Figure 5). There was significant heterogeneity of results across studies $\left(\mathrm{p}<0.01 ; I^{2}=87.03\right)$. However, sensitivity tests did not reveal any individual study that exerted influence on the overall estimate. There was no evidence of publication bias $(p=0.76)$. The difference between the pooled prevalence of UAI with older MSM was significantly higher than their younger counterparts (79.8\% vs. $61.7 \%$, Chi-square value $=238.2, \mathrm{p}<0.001)$.

\section{Discussion}

To our knowledge, this meta-analysis provides the first quantitatively synthesized estimates of the prevalence rates of HIV, syphilis and UAI among older MSM in China. The summarized estimates were HIV (11.6\%; 95\% CI: $8.0 \%$ 16.6\%), syphilis (23.0\%; 95\% CI: $15.8 \%-32.3 \%)$ and UAI (79.5\%; 95\% CI: 72.7\%-84.9\%). Our meta-analysis also demonstrated that the trend of HIV prevalence among older MSM has substantially increased in the past decade. 
Table 2 Stratified meta-analyses of HIV prevalence among older MSM of China

\begin{tabular}{|c|c|c|c|c|}
\hline \multirow[t]{2}{*}{ Subgroups } & \multirow[t]{2}{*}{ Prevalence $\%(95 \% \mathrm{Cl})$} & \multirow[t]{2}{*}{ No of studies } & \multicolumn{2}{|c|}{ Heterogeneity } \\
\hline & & & $\mathrm{I}^{2} \%$ & $\overline{p \text { Value }}$ \\
\hline \multicolumn{5}{|l|}{ Sample size } \\
\hline$>200$ & $7.2 \%(5.4 \%-9.5 \%)$ & 3 & 70.67 & $<0.05$ \\
\hline$\leq 200$ & $13.6 \%(8.4 \%-21.2 \%)$ & 17 & 55.86 & $<0.05$ \\
\hline \multicolumn{5}{|l|}{ Study year } \\
\hline $2003-2007$ & $9.9 \%(2.7 \%-30.6 \%)$ & 4 & 89.30 & $<0.05$ \\
\hline 2008-2009 & $12.1 \%(6.8 \%-20.5 \%)$ & 9 & 81.04 & $<0.05$ \\
\hline $2010-2011$ & $13.6 \%(8.2 \%-21.8 \%)$ & 7 & - & $>0.05$ \\
\hline \multicolumn{5}{|l|}{ Study location } \\
\hline East China & $13.0 \%(6.8 \%-23.4 \%)$ & 8 & 32.12 & $>0.05$ \\
\hline Nationwide & $7.2 \%(5.4 \%-9.5 \%)$ & 3 & 70.67 & $<0.05$ \\
\hline North China & $14.7 \%(2.7 \%-51.5 \%)$ & 2 & 70.91 & $>0.05$ \\
\hline South China & $12.3 \%(3.3 \%-36.6 \%)$ & 3 & 35.25 & $>0.05$ \\
\hline Southwest & $16.4 \%(2.8 \%-57.4 \%)$ & 3 & 83.90 & $<0.05$ \\
\hline West China & $9.5 \%(2.4 \%-31.1 \%)$ & 1 & - & $<0.05$ \\
\hline \multicolumn{5}{|l|}{ Recruitment settings } \\
\hline Medical settings & $7.9 \%(6.7 \%-9.3 \%)$ & 11 & - & $>0.05$ \\
\hline Multiple gay venues or events & $17.4 \%(7.6 \%-35.0 \%)$ & 9 & 89.20 & $<0.05$ \\
\hline \multicolumn{5}{|l|}{ Sampling method } \\
\hline Mixed & $15.5 \%(8.0 \%-27.8 \%)$ & 10 & 52.88 & $<0.05$ \\
\hline RDS & $8.0 \%(6.7 \%-9.6 \%)$ & 2 & 2.98 & $>0.05$ \\
\hline Snowballing & $9.8 \%(4.7 \%-19.2 \%)$ & 8 & 78.12 & $<0.05$ \\
\hline
\end{tabular}

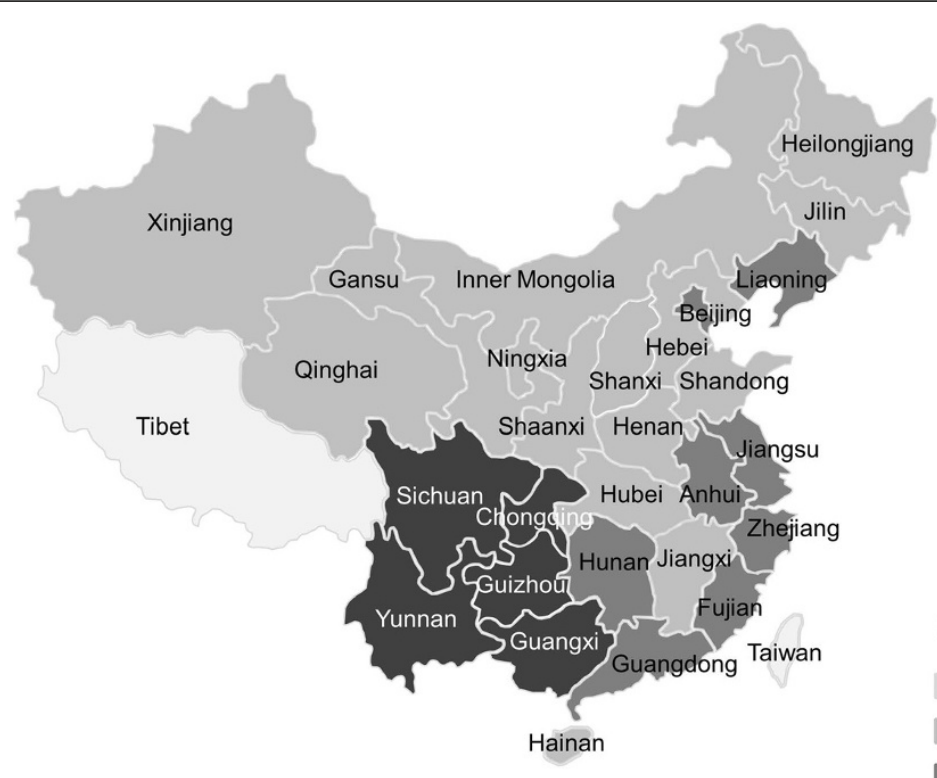

Prevalence estimate(\%)

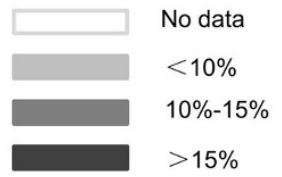

Figure 4 The regional distribution of pooled prevalence of HIV among older MSM in China. Created by: YZL. Generated by: Microsoft Office PowerPoint. 


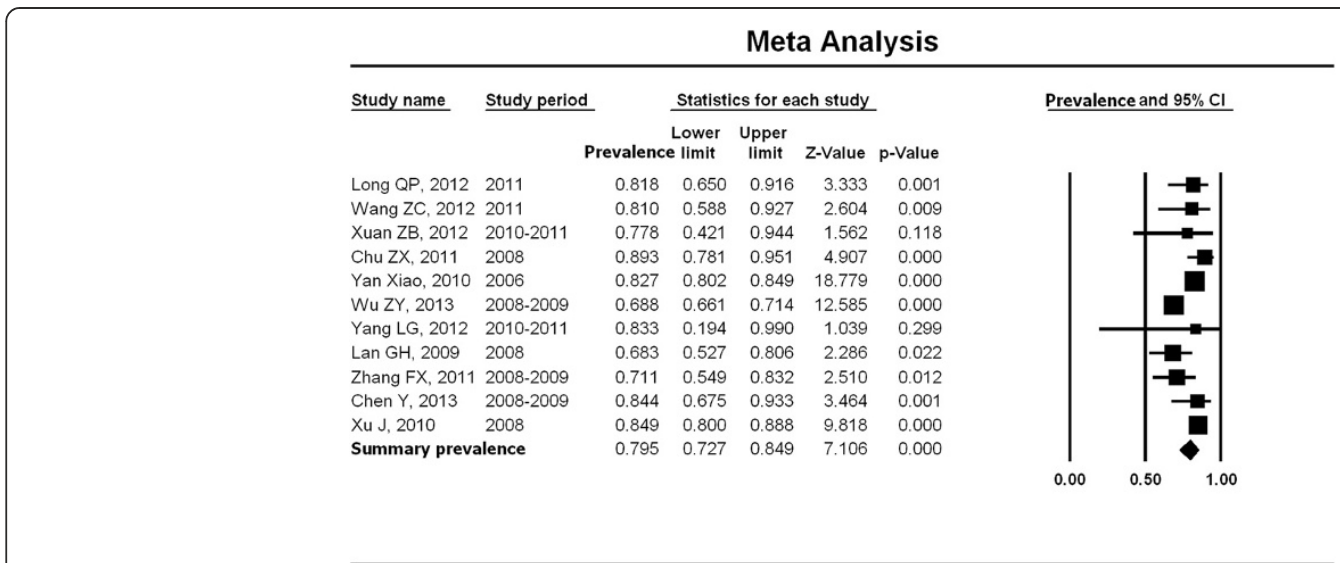

Figure 5 Meta-analysis of UAl prevalence among older MSM in China. Figure 5 shows unadjusted UAI prevalence estimates (boxes) with 95\% confidence limits (bars) for each study selected; pooled prevalence estimates are represented as diamonds in this plot.

Besides, our analysis also provided evidence contradictory to a widely accepted assumption that young MSM was at higher HIV risk than older MSM $[35,36]$. The overall and stratified estimates provide useful formation for epidemiologic modeling of HIV transmission among older MSM and for allocating resource for targeted prevention interventions.

HIV prevalence was higher among older MSM than their younger counterparts ( $11.6 \%$ vs. $5.2 \%)$, and is higher than average prevalence (4.9\%) among nearly 50,000 MSM in a nationwide survey during 2008 and 2009 [2], and is also higher than the average prevalence among national sentinel sample (6.3\% for HIV, and $10 \%$ for syphilis) [3]. Firstly, it is possible that older MSM had longer durations of exposure to HIV source. Secondly, HIV testing as increased HIV prevalence may be to some extent due to increase testing rate since HIV testing among Chinese MSM has been increasing over the decade [37], then antiretroviral treatment could also contribute to a higher prevalence among older MSM as they just age with their infection. In addition, UAI among older MSM was also more common than their younger counterparts, which might partly explain the higher HIV prevalence among older MSM. HIV prevalence among older MSM in China is higher than older MSM in European countries such as Sweden (3.8\%) and Germany (2.6\%), but was much lower than those in four major cities in the United States (19\%) [38,39].

Syphilis infection is a good surrogate for high risk sexual behaviors. A high prevalence of syphilis (23.0\%) among Chinese older MSM suggested that these men may have heavily engaged in unprotected sex, which could facilitate HIV spread in this group, and screening and treating syphilis could be an effective way prompting HIV detection [40]. Syphilis itself would also act as a cofactor for HIV acquisition [41-44]. Due to the mode of sexual transmission, single public health intervention campaign can be designed with the objectives of preventing HIV and preventing syphilis. Although UAI is common among older MSM, HIV has not yet reached the high level as syphilis. We interpret this as a significant opportunity to save lives through increasing public health intervention efforts [2].

We also found that a sizeable percentage $(79.5 \%)$ of older MSM had engaged in UAI with male partners. This is an important public health concern, given the high prevalence of HIV and STIs among older MSM in China. The UAI prevalence was higher among older MSM than among their younger counterparts, which was generally consistent with several recent studies in China $[11,45]$, but it is contradictory to the studies in the United States [46].

Subgroup analysis showed the highest HIV infection rate in southwest China (16.4\%), which was consistent with prior studies $[47,48]$.

Older MSM living with HIV may bring more medical challenges. The elders are more vulnerable to side effects of antiretroviral drugs [49]. Aged people are facing problems of diabetes and heart disease, whereas research suggests that antiretroviral drugs contribute to high cholesterol levels and hamper insulin production, thus increasing the risk of health problems [50]. Besides, significant HIV epidemic among older MSM is not only a medical issue but also a social problem. Due to traditional Chinese values, the majority was married, but they continued engaging in extramarital sex with their male partners. Studies have shown that they are less likely to take precautions [51], even though they know HIV risk from unprotected sex [52], because they have misconception that HIV/AIDS is a disease of young people [53]. What is worse, some of them hold opinion perception that the latent time from infection to development of AIDS could be up to ten years, and they may die of other diseases, so they do not even care about HIV/AIDS [54]. Little HIV prevention work has focused on older MSM, partly due to the widespread belief that older MSM are not sexually active [46]. Our analysis provides strong evidence of the need for interventions in this 
subgroup. Formative work is needed to determine appropriate approaches for this population. Some of the older MSM are likely to engage in bisexual behaviors, which may play a bridging role in the spread of HIV and other sexual transmitted diseases from this high-risk group to the general population [55]. Moreover, the alarming spread of HIV among older MSM is fuelled by an ongoing, persistent stigma against homosexuality in China [56]. This may lead to a result that older MSM hide their sexual identity, thereby increasing the difficulty of reaching them by prevention interventions. It is critical to reduce stigma against homosexuality and mitigating discrimination against homosexuals among the general population.

Our meta-analytic findings should be viewed within the context of the methodological limitations of the primary studies. Our findings are based on cross-sectional data, which provide snap shots of prevalence of HIV, syphilis, and UAI at the time of data collection. The included studies are heterogeneous in term of sample size; recruitment setting, sampling method, study year, and study location. For example, some studies recruited participants through RDS or snowball sampling rather than random sampling, and these sampling approaches may have selection bias [40]; More studies were conducted in east China, and few in west China. Therefore, the summary estimated may not represent the national sample. Small sample size may also restrict the statistical power in subgroup analyses, there are very small sample sizes of many studies and probably also bias in some studies. Though no major publication bias was indicated in the meta-analysis, the possibility of publication bias could not be fully excluded. In the stratified analyses, heterogeneity between studies was reduced, suggesting that the varied data collection method, recruitment setting, sample size, study time and study locations may account for the inconsistency among studies. A higher prevalence of HIV infection was found among studies with sample sizes $\leq 200$ compared with sample sizes $>200$, and this may suggest the prevalence may be overestimated in the studies with a smaller sample size.

\section{Conclusion}

Our meta analysis provided important findings on HIV and syphilis epidemics and UAI among older MSM in China. These findings are contradictory to the widespread perception that young MSM are at the highest risk of HIV infection. Older MSM have been ignored in HIV/STI prevention intervention programs, and more focus should be given to this neglected and hard-toreach subgroup in future HIV interventions. Further large-scale epidemiological investigations, with standard sampling methods and adequate power, should be conducted to gain a more precise estimate of the status of the HIV and other sexually transmitted infection epidemics among older MSM in China.

\section{Additional file}

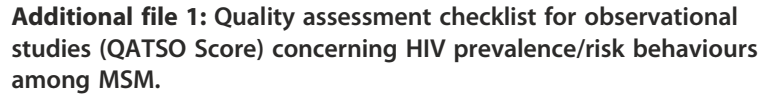

\section{Abbreviations}

HIV: Human immunodeficiency virus; AIDS: Acquired immune deficiency syndrome; UAI: Unprotected anal intercourse; MSM: Men who have sex with men; STI: Sexually transmitted infection.

\section{Competing interests}

The authors declare that they have no competing interests.

\section{Authors' contributions}

Conceived and designed the experiments: YZL HS JJX. Performed the experiments: YZL BXY. Analyzed the data: YZL JMZ QHH ZXC SYL. Contributed reagents/materials/analysis tools: HS. Wrote the paper: YZL JJX. Revised the language: BXY JZ HZQ. Revised the article: HS YJJ WQG HZQ. All authors read and approved the final manuscript.

\section{Acknowledgements}

This study was supported by Grants from the Mega-Projects of National Science Research for the 12th Five-Year Plan (2012ZX10001006); National Nature Science Foundation of China (81001291); Innovation Team Development Program 2012 of the Ministry of Education. The authors appreciate all the researchers whose articles were included in the study.

\section{Author details}

${ }^{1}$ Key Laboratory of AIDS Immunology of National Health and Family Planning Commission, Department of Laboratory Medicine, The First Affiliated Hospital, China Medical University, Shenyang, Liaoning, China. ${ }^{2}$ Collaborative Innovation Center for Diagnosis and Treatment of Infectious Diseases, Hangzhou, China. ${ }^{3}$ Vanderbilt Institute for Global Health, Vanderbilt University, Nashville, TN, USA. ${ }^{4}$ Division of Epidemiology, Department of Medicine, Vanderbilt University Medical Center, Nashville, TN, USA. ${ }^{5}$ Department of Finance, Business College, Washington State University, Pullman, WA, USA. ${ }^{6}$ Department of Clinical Epidemiology and Evidence-based Medicine, The First Affiliated Hospital, China Medical University, Shenyang, Liaoning, China.

Received: 3 April 2014 Accepted: 16 September 2014

Published: 6 October 2014

\section{References}

1. Zhang L, Chow EP, Jing J, Zhuang X, Li X, He M: HIV prevalence in China: integration of surveillance data and a systematic review. Lancet Infect Dis 2013, 13:955-63.

2. Wu Z, Xu J, Liu E, Mao Y, Xiao Y, Sun X: HIV and syphilis prevalence among men who have sex with men: a cross-sectional survey of 61 cities in China. Clin Infect Dis 2013, 57:298-309.

3. State Council AIDS Working Committee Office: A joint assessment of HIV/AIDS prevention, treatment and care in China. [Available: http://www.chinaids.org.cn]

4. Ministry of Health, People's Republic of China: 2012 China AIDS Respons Progress Report. [http://www.unaids.org.cn/cn/index/page.asp? id =197\&class=2\&classname=China's+Epidemic+\%26+Response+ $]$

5. China National Committee On Ageing: China's aging population will exceed 200 million at the end of 2013. [http://finance.chinanews.com/jk/ 2012/09-05/4160829.shtml]

6. Wu YS: Annual Report on Development of the Cause of Aging. Beijing, China: Social Science Academic Press; 2013.

7. Zou H, Wu Z, Yu J, Li M, Ablimit M, Li F: Sexual risk behaviors and HIV infection among men who have sex with men who use the internet in Beijing and Urumqi, China. J Acquir Immune Defic Syndr 2010, 53:81.

8. Biyun Q, Xi C, Yujun Y, Qi L, Jie L, Xianguo S: Qualitative study on HIV-related information in senile HIV-positives in Hunan. China Trop Med 2012, 12:677-678,687.

9. Guangzi Q, Yaqin P: Survey of cognitive attitude of medium-elderly men to AIDS in area with high incidence of AIDS. Chin J Gerontol 2012, 32:784-785. 
10. Jie L, Xi C, Biyun T, Wenbo H, Wenjuan Z: Investigation of HIV-related risk factors among elderly HIV-positive patients. Pract Prev Med 2010, 17:227-229.

11. Zhu M, Zhang B, Li X, Shi T, Wu S: The Relationship of Age and High Risk Behaviors Related to HIV, AIDS among Men Who Have Sex with Men in China. Chin J Drmatol 2004, 37:635-637.

12. Moher D, Liberati A, Tetzlaff J, Altman DG: Preferred reporting items for systematic reviews and meta-analyses: the PRISMA statement. Int I Surg 2010, 8:336-341.

13. Wong WC, Cheung CS, Hart GJ: Development of a quality assessment tool for systematic reviews of observational studies (QATSO) of HIV prevalence in men having sex with men and associated risk behaviours. Emerg Themes Epidemiol 2008, 5:23.

14. Lipsey MW, Wilson DB: Practical Meta-analysis. Thousand Oaks, California: Sage Publications; 2001.

15. Hunter JE, Schmidt FL, Jackson GB: Meta-analysis: Cumulating research findings across studies. Thousand Oaks, California: Sage Publications; 1982

16. Aibing $M$, An Xiaojing $Y$, Huifang $Z Y$, Zhimin $Y$ : The analysis of 78 cases with HIV infection test results among men who have sex with men in Yunnan. Chin J AIDS STD 2007, 13:60-61.

17. Xi S: Study on AIDS Prevention knowledge, behavioral characteristics, HIV infection and its influencing factors among men who have sex with men(MSM)in Hangzhou City. In PhD thesis. Zhejiang University: Department of Social Medicine \& Health Management; 2011.

18. Qingping L, Peilan Q, Zhenhao H, Jian C, Weilv W: Survey on a Cluster of HIV Infection Among MSMs in a Club and Exploration of its Influencing Factors. Pract Prev Med 2012, 19:1903-1906.

19. Zhou JB, Hao C, Huan XP, Wang JT, Zhen S: HIV and syphilis infections among men who have sex with men. Chin J Public Health 2012, 28:1031.

20. Wang Z: A survey of HIV infections and related factors among men who have sex with men in Xining Qinghai province. In PhD thesis. Shanxi Medical University; 2012.

21. He Q, Wang Y, Lin P, Liu Y, Yang F, Fu X: Potential bridges for HIV infection to men who have sex with men in Guangzhou, China. AIDS Behav 2006, 10:17-23.

22. Zhenxing C, Ma Ning $X$, Junjie ZM, Qinghai $H$, Zhang J: HIV prevalence and its associated factors among 2074 men who have sex with men (MSM) in Liaoning province, China. Chin J Public Health 2011, 27:967-969.

23. Xiao Y, Sun J, Li C, Lu F, Allen KL, Vermund SH: Prevalence and correlates of HIV and syphilis infections among men who have sex with men in seven provinces in China with historically low HIV prevalence. JAIDS 2010, 53:S66-S73.

24. Yanqiu Z, Jie G, Kaikan G, Hui W, Xiaolong M: Testing of HIV and Syphilis and Investigation of Risk Factors Among 416 MSM. Chinese Prim Health Care 2012, 26:11

25. Luguang $Y$, Xiuping $S$, Xinping D, Liang S, Duan $Y$ : Study On AIDS Knowledge, Behavior And HIV infection Among MSM In Fuyang Of Anhui Province. Anhui J Prev Med 2012, 18:22-23.

26. Gunghua L, Wei L, Qiuying Z, Fuxiong L, Yuman H: Surveillance of HIV Infection Among Different Aged Men Having Sex With Men in Guangxi. J Prev Med Inf 2009, 25:608-611.

27. Feng F, Wang Z, Huang S, LV J, Lin Z: Investigation on AIDS knowledge, attitude and practice characteristics of MSM group and HIV/syphilis infection situation. Mod Prev Med 2009, 36:2902-2903.

28. Fuxin Z, Xiuping Z, Xiaoping C, Yanqiu Z, Guangjie C: Sero-prevalence and correlates of HIV infection among men who have sex with men in Suzhou. Chin J Dis Control Prev 2011, 15:1031-1034.

29. Rui L, Wei T, Zhijie Z: Survey on HIV Infection and Related Factors and High Risk Behaviors among Men who Have Sex with Men in Dalian City, 2009. Prev Med Trib 2010, 16:1127-1129.

30. Yang C, Limei S, Yongming Y, Jian Z, Fei Y: HIV/AIDS epidemic among 1045 MSMs and the associated factors in two cities of Guizhou. Chin J AIDS STD 2013, 06:402-405+412.

31. Zhimin N, Li Jian $X$, Dange $Z Y$, Ting C: An Investigation on the Infection Rate of Syphilis and HIV among MSM Population from Public Bath. Zhejiang Prev Med 2011, 23:14-16.

32. Xu J, Han D, Liu Z, Ma X, Wang L, Xu J: The prevalence of HIV infection and the risk factors among MSM in 4 cities. China Chin J Prev Med 2010, 44:975-980
33. Luxiang Z, Jincai Q, Qianjin C: The surveillance of HIV infection and sexual behaviors amon MSM in Longyan, 2010-2011. Strait J Prev Med 2012, 18:34-35.

34. Zhoubin X, Huimin H, Yun H, Hui T, Zongxia Z: Sexual characteristics and HIV and syphilis infections among 98 MSM in Yangpu District, Shanghai. Chin J Dis Control Prev 2012, 16:1028-1031

35. Meiying C, Chenghua G, Liping D, Yihai J, Fan W: Sexual behaviors and $\mathrm{HIV} /$ syphilis infection among 435 men who have sex with men. South China J Prev Med 2010, 36:28-30.

36. Xiaoyan M, Willi MF, Qiyun Z, Jinkou Z, Xiong H: Analysis on trend of HIV prevalence among men having sex wtih men. Chin J Public Health 2007, 23:1352-1354

37. Zou H, Hu N, Xin Q, Beck J: HIV testing among men who have sex with men in China: a systematic review and meta-analysis. AIDS Behav 2012, 16:1717-28.

38. Catania JA, Osmond D, Stall RD, Pollack L, Paul JP, Blower S: The continuing HIV epidemic among men who have sex with men. Am J Public Health 2001, 91:907.

39. Marcus U, Hickson F, Weatherburn P, Schmidt AJ, the EMIS network: Age biases in a large HIV and sexual behaviour-related internet survey among MSM. BMC Public Health 2013, 13:1-11.

40. Gao L, Zhang L, Jin Q: Meta-analysis: prevalence of HIV infection and syphilis among MSM in China. Sex Transm Infect 2009, 85:354-358.

41. Weinstock H, Sweeney S, Satten GA, Gwinn M: HIV seroincidence and risk factors among patients repeatedly tested for HIV attending sexually transmitted disease clinics in the United States, 1991 to 1996. J Acquir Immune Defic Syndr Hum Retrovirol 1998, 19:506-512

42. Otten MW Jr, Zaidi AA, Peterman TA, Rolfs RT, Witte JJ: High rate of HIV seroconversion among patients attending urban sexually transmitted disease clinics. AIDS 1994, 8:549-53.

43. Ruan Y, Li D, Li X, Qian H, Shi W, Zhang X: Relationship between syphilis and HIV infections among men who have sex with men in Beijing, China. Sex Transm Dis 2007, 34:592-597.

44. Xu JJ, Zhang M, Brown K, Reilly K, Wang H, Hu QH: Syphilis and HIV seroconversion among a 12-month prospective cohort of men who have sex with men in Shenyang, China. Sex Transm Dis 2010, 37:432-439.

45. Wang Yi X, Jie LZ, Guanggui Z, Liulin L: The use of condoms among MSM and the influencing factors. Chin J Dis Control Prev 2012, 16:133-136.

46. Dolcini MM, Catania JA, Stall RD, Pollack L: The HIV epidemic among older men who have sex with men. JAIDS 2003, 33:S115-S121.

47. Li Y, Xu J, Reilly KH, Zhang J, Wei H, Jiang Y: Prevalence of HIV and syphilis infection among high school and college student MSM in China: a systematic review and meta-analysis. PLoS One 2013, 8:e69137.

48. Chow EP, Wilson DP, Zhang J, Jing J, Zhang L: Human immunodeficiency virus prevalence is increasing among men who have sex with men in China: findings from a review and meta-analysis. Sex Transm Dis 2011, 38:845-57.

49. Tesfaye DY, Kinde S, Medhin G, Megerssa YC, Tadewos A, Tadesse E: Burden of metabolic syndrome among HIV-infected patients in Southern Ethiopia. Diabetes Metab Syndr 2014, 8:102-7.

50. Vigouroux C, Gharakhanian S, Salhi Y, Nguyen TH, Chevenne D, Capeau J: Diabetes, insulin resistance and dyslipidaemia in lipodystrophic HIV-infected patients on highly active antiretroviral therapy (HAART). Diabetes Metab 1999, 25:225-32.

51. Fang W: Increasing HIV infections among MSM and the elderly. [http://news.sina.com.cn/c/2012-11-30/031925695231.shtml]

52. Crepaz N, Marks G, Liau A, Mullins MM, Aupont LW, Marshall KJ: Prevalence of unprotected anal intercourse among HIV-diagnosed MSM in the United States: a meta-analysis. AIDS 2009, 23:1617-1629.

53. Qin X, Fan L, Hao Z, Ye Y: Analysis of HIV/AIDS Prevalence of the Older Persons in China. Population \& Economics 2005, 6:1-5.

54. Li Q: The rapid prevalence of HIV among older men and the diversification among AIDS patients in China. [http://news.xinhuanet.com/2012-08/23/c_ 112814803.htm]

55. Cao Z, Zhang H, Shen M, Wang J, Xu J: Prevalence of HIV infection and sexual behaviors with both men and women among currently married men who have sex with men. Chin J Epidemiol 2012, 33:488-491.

56. Shang H, Xu J, Han X, Li JS, Arledge KC, Zhang L: HIV prevention: Bring safe sex to China. Nature 2012, 485:576-577.

doi:10.1186/1471-2334-14-531

Cite this article as: Li et al:: High prevalence of HIV infection and unprotected anal intercourse among older men who have sex with men in China: a systematic review and meta-analysis. BMC Infectious Diseases 2014 14:531. 